\title{
Singularities of the Scattering Kernel for Two Convex Obstacles
}

\author{
By \\ Shin-ichi NAKAMURA* \\ Dedicated to Professor Takao Kakıta for his sixtieth birthday
}

\section{§ 1. Introduction}

We consider the scattering problem for the wave equation by two finite obstacles. Let $\mathcal{O}$ be a compact set in $\boldsymbol{R}^{n}(n \geqq 2)$, and assume that $\Omega=\boldsymbol{R}^{n}-\mathcal{O}$ is connected. Consider the following problem

$$
\left\{\begin{aligned}
\square u(t, x)=\left(\partial_{t}^{2}-\Delta_{x}\right) u(t, x)=0 & & \text { in } \boldsymbol{R}^{1} \times \Omega, \\
u(t, x)=0 & & \text { on } \boldsymbol{R}^{1} \times \partial \Omega, \\
u(0, x)=f_{1}(x) & & \text { on } \Omega, \\
\partial_{t} u(0, x)=f_{2}(x) & & \text { on } \Omega .
\end{aligned}\right.
$$

Denote by $k_{-}(s, \omega)\left(k_{+}(s, \omega)\right) \in L^{2}\left(\boldsymbol{R}^{1} \times S^{n-1}\right)$ the incoming (outgoing) translation representation of the initial data $\left(f_{1}, f_{2}\right)$. The scattering operator $S: k_{-} \rightarrow k_{+}$has a distribution kernel $S(s, \theta, \omega)$ called the scattering kernel (cf. Lax and Phillips [6], [7]).

Recently several authors have studied on the relation between $\mathcal{O}$ and $S(s, \theta, \omega)$. Soga [14] and Yamamoto [17] have characterized the convexity of $\mathcal{O}$ in terms of the singularities of $S(s,-\omega, \omega)$ as follows:

$$
\begin{aligned}
& \mathcal{O} \text { is convex if and only if sing supp } S(\cdot,-\omega, \omega) \text { has only } \\
& \text { one point for any } \omega \in S^{n-1} \text {. }
\end{aligned}
$$

The purpose of this paper is to study the location of sing supp $S(\cdot,-\omega, \omega)$ when $\mathcal{O}$ consists of two convex obstacles $\mathcal{O}_{1}$ and $\mathcal{O}_{2}$. Set $r_{i}(\omega)=\min _{x \in \mathcal{O}_{2}} x \cdot \omega(i=1,2)$. Suppose that $\mathcal{O}_{1} \cap \mathcal{O}_{2}=\phi$. The first main results is the following:

Communicated by S. Matsuura, July 29, 1987.

* Department of Mathematics, School of Science and Engineering, Waseda University. 
Theorem 1. Let $\omega \in S^{n-1}$ satisfy

$$
\mathcal{O}_{1} \cap\left\{x+l \omega: x \in \mathcal{O}_{2}, l \in \boldsymbol{R}^{1}\right\}=\phi \text {. }
$$

Then there holds

sing supp $S(\cdot,-\omega, \omega) \cap\left[\min _{i=1,2}\left(-2 r_{i}(\omega)\right),+\infty\right)=\left\{-2 r_{1}(\omega),-2 r_{2}\right.$ $(\omega)\}$.

For the more restricted $\omega$, we can know precisely sing supp $S(\cdot,-\omega, \omega)$ which is included in $\left(-\infty, \min _{i=1,2}\left(-2 r_{i}(\omega)\right)\right)$. Let $x_{0} \in P=\left\{x: x \cdot \omega=\min _{i=1,2} r_{i}(\omega)-1\right\}$, and consider the broken ray starting at $x_{0}$ in the direction $\omega$ at the law of the geometrical optics. Suppose that this ray is reflected once at each points $x_{1}, \cdots, x_{m}$ on the boundary and returns to the point $x_{m+1}$ on the hyperplane $P$ in the direction $-\omega$. To those points we assign the numbers

$$
s_{m}^{i}=\sum_{j=1}^{m+1}\left|x_{j-1}-x_{j}\right|-2 \quad x_{1} \in \partial \mathcal{O}_{i}(i=1,2) .
$$

Theorem 2. Let $\omega \in S^{n-1}$ satisfy the same assumption as in Theorem 1. Then the following statements hold:

(I) For any positive integer $m$ there exist the broken rays and a constant $G_{1}>0$ independent of $m$ and $i$ such that

(1.4) $s_{m}^{i}-\operatorname{dist}\left(P, x_{1}\right)-\operatorname{dist}\left(P, x_{m}\right)-(m-1) \operatorname{dist}\left(\mathcal{O}_{1}, \mathcal{O}_{2}\right) \leqq C_{1}$.

(II) If

$$
\operatorname{dist}\left(\mathcal{O}_{1}, \mathcal{O}_{2}\right)>\dot{4} \max _{i=1,2} \operatorname{diam} \mathcal{O}_{i}+C_{1}
$$

and

$$
\left|r_{1}(\omega)-r_{2}(\omega)\right|<\max _{i=1,2} \operatorname{diam} \mathcal{O}_{i},
$$

then we have

(i) $\min _{i=1.2} s_{m+1}^{i}>\max _{i=1,2} s_{m}^{i} \quad$ for $m \geqq 1$,

(ii) sing $\operatorname{supp} S(\cdot,-\omega, \omega)=\left\{-2 \min _{i=1,2} r_{i}(\omega)-s_{m}^{i}\right\}_{\substack{i=1,2 \\ m=1,2, \ldots}}$.

(III) Let $\operatorname{dist}\left(\mathcal{O}_{1}, \mathcal{O}_{2}\right)$ and $\omega$ satisfy the assumption in (II). And further if there exists the only one pair of points $\left(a_{1}, a_{2}\right) \in \partial \mathcal{O}_{1} \times \partial \mathcal{O}_{2}$ such that $\left|a_{1}-a_{2}\right|=\operatorname{dist}\left(\mathcal{O}_{1}, \mathcal{O}_{2}\right)$, then the following equalities hold:

(iii) $\lim _{m \rightarrow+\infty}\left(s_{m+2}^{i}-s_{m}^{i}\right)=2 \operatorname{dist}\left(\mathcal{O}_{1}, \mathcal{O}_{2}\right) \quad(i=1,2)$,

(iv) $\lim _{m \rightarrow+\infty}\left\{s_{2 m}^{i}-2^{-1}\left(s_{2 m-1}^{1}+s_{2 m-1}^{2}\right)\right\}=\operatorname{dist}\left(\mathcal{O}_{1}, \mathcal{O}_{2}\right)(i=1,2)$. 
In case each $\mathcal{O}_{i}$ is a ball in $\boldsymbol{R}^{2}$ or $\boldsymbol{R}^{3}$, these theorems corresponding to Theorem 1 and Theorem 2 have been proved by Nakamura and Soga [10] under the slightly different assumptions. The main tasks in the proof of Theorem 2 are to show that there exist actually the broken rays with the properties (i), (ii) and (iv) stated in Theorem 2. In [10] they have been proved the existence of such rays in the case of two disjoint disks in $\boldsymbol{R}^{2}$, and that the proof in $\boldsymbol{R}^{3}$ can be reduced to that in $\boldsymbol{R}^{2}$ when both $\mathcal{O}_{1}$ and $\mathcal{O}_{2}$ are balls. However to the present case we cannot apply the method employed in $[10]$.

Our proof is based on the following thoughts. Let $\omega$ be the direction given in Theorem 1. Suppose a plane wave propagates in the direction $\omega$ and hits a convex obstacle $\mathcal{O}_{1}$. Then the wave front reflected by $\mathcal{O}_{1}$ will be have no focal points and that there will be at least one broken ray reflected in the direction $-\omega$. Suppose the wave front reflected by $\mathcal{O}_{1}$ hits $\mathcal{O}_{2}$ subsequently and is reflected by $\mathcal{O}_{2}$. Then it also has no focal points and there exists at least one reflected ray with the direction $-\omega$ again. Using the precise investigation of the convexity property concerned with the incident wave front, the reflected one and the obstacle (Lemma 2.1), we can check that this process is successively true. For proving the property (i) in Theorem 2 it is essential to use the estimate (1.4). The estimate of the type (1.4) is proved in [10] based on the fact the Gaussian curvature of the boundary $\partial \Omega$ does not vanish anywhere. However, this is not our case. Actually we derive (1.4) by estimating more precisely the difference $\left|x_{j}-x_{\jmath+1}\right|-\operatorname{dist}\left(\mathcal{O}_{1}, \mathcal{O}_{2}\right)$ (Lemma 2.6).

After completing this work, the author is informed that Petkov and Stojanov [12] (without proofs) have extended our results [10] to the case for several strictly disjoint obstacles in $\boldsymbol{R}^{3}$ under some assumptions. But it seems to the author that all our results cannot be obtained from theirs, especially the properties (i) and (iv) stated in Theorem 2.

\section{§2. Properties of the Broken Rays}

First, we define the broken rays according to the law of the geometrical optics. For $x \in \partial \Omega$, denote by $\nu(x)$ the unit inward normal 
defined at points of the boundary. We suppose that $\left\{x=x_{0}+l \xi_{0} ; l>\right.$ $0\} \cap \partial \Omega \neq \phi$ for $x_{0} \in \Omega$ and $\xi_{0} \in S^{n-1}$. We define $l_{j_{-1}}, x_{j}$ and $\xi_{j}$ successively by

$$
\begin{aligned}
& l_{j-1}=\inf \left\{l>0 ; \quad x_{j-1}+l \xi_{j-1} \in \partial \Omega\right\} . \\
& x_{j}=x_{j-1}+l_{j-1} \xi_{j-1}, \\
& \xi_{j}=\xi_{j-1}-2\left(\xi_{j-1} \cdot \nu\left(x_{j}\right)\right) \nu\left(x_{\jmath}\right),
\end{aligned}
$$

where $l_{j-1}=\infty$ when $x_{j-1}+l \xi_{j-1} \notin \partial \Omega$ for any $l>0$. Assuming that these $\left\{l_{j}\right\},\left\{x_{j}\right\}$ and $\left\{\xi_{j}\right\}$ are well-defined, we call the set

$$
L\left(x_{0}, \xi_{0}\right)=\bigcup_{j}\left\{x=x_{j}+l \xi_{j} ; 0 \leqq l \leqq l_{j}\right\}
$$

the broken rays starting at $x_{0}$ in the direction $\xi_{0}$, and $\left\{x_{j}\right\}$ the reflection points. When there exists an integer $m \geqq 1$ such that $\left\{x=x_{m}+l \xi_{m} ; l>0\right\} \cap \partial \Omega=\phi$, we set

$$
\begin{aligned}
& \#_{\text {ref } L\left(x_{0}, \xi_{0}\right)}=m, \\
& \operatorname{dir}_{\infty} L\left(x_{0}, \xi_{0}\right)=\xi_{m} .
\end{aligned}
$$

The purpose in this section is to show some theorems which play an important role in the proof of Theorem 2 in Introduction.

Theorem 2.1. Let $\omega \in S^{n-1}$ satisfy

$$
\mathcal{O}_{1} \cap\left\{x+l \omega: x \in \mathcal{O}_{2}, l \in \boldsymbol{R}^{1}\right\}=\phi .
$$

Then for any positive integer $m$, there exist a broken ray $L^{i}\left(x_{0}, \omega\right) \quad(i=1$, 2) such that

$$
\begin{aligned}
& \text { (i) } x_{0} \text { is on the plane } P=\left\{x: x \cdot \omega=\min _{i=1,2} r_{i}(\omega)-1\right\} \text {, } \\
& \text { (ii) the first reflection point } x_{1} \text { belongs to } \mathcal{O}_{i}, \\
& \text { (iii) }{ }^{\text {ref }} L^{i}\left(x_{0}, \omega\right)=m \text {, } \\
& \text { (iv) } \operatorname{dir}_{\infty} L^{i}\left(x_{0}, \omega\right)=-\omega \text {. }
\end{aligned}
$$

Furthermore, if there exist the broken ray $L^{i}\left(x_{0}, \omega\right)$ and $L^{i}\left(y_{0}, \omega\right)$ satisfying the above conditions (i) (iv), then we have

$$
\sum_{j=1}^{m+1}\left|x_{j-1}-x_{j}\right|=\sum_{j=1}^{m+1}\left|y_{j-1}-y_{j}\right|
$$

where $\left\{x_{j}\right\}$ (resp. $\left\{y_{j}\right\}$ ) are the reflection points of the broken ray $L^{i}\left(x_{0}, \omega\right) \quad\left(\operatorname{resp} . L^{i}\left(y_{0}, \omega\right)\right)$.

First, we study the convexity of the surface. Let $M$ be a smooth hypersurface in $\boldsymbol{R}^{n}$. Let $v$ be any tangent vector at $x \in M$. Pick any 
curve $x=x(t)$ through $x$ so that $d x /\left.d t\right|_{t=0}=v$. Then we define the operator $A$ (called the shape operator) as follows:

$$
d \nu(x(t)) /\left.d t\right|_{t=0}=A v
$$

where $\nu(x)$ is a unit vector at $x$ normal to $M$. The following properties of $A$ are well known (cf. $\S 8.2$ in [1]).

(i) $A$ is well-defined independently of the choice of the curve $x(t)$ so long as it has the prescribed tangent vector $v$ at $t=0$.

(ii) $A$ is the self-adjoint linear operator on the tangent space $T_{x} M$ at $x$.

(iii) The hypersurface $M$ is convex if and only if for any $x \in M A$ is positive (or negative) semi-definite.

(iv) Let $\left\{x=\Phi(\sigma) ; \sigma \in U \subseteq \boldsymbol{R}^{n-1}\right\}$ be a local coordinate system of $M$ such that $x^{0}=\Phi(0)$. Then we have the following representation of $A$ at $x^{0}$ with respect to the basis $\partial \Phi / \partial \sigma_{k}(0)(k=1,2$, $\cdots, n-1)$ :

$$
\left(\partial(\nu \circ \Phi) / \partial \sigma_{j}(0) \cdot \partial \Phi / \partial \sigma_{k}(0)\right)_{j, k=1,2, \cdots, n-1},
$$

where $\left(a_{\jmath k}\right)_{j, k=1,2, \cdots, n-1}$ is a $(n-1) \times(n-1)$ matrix.

We will use later the notation ${ }^{t}\left(a_{j k}\right)_{j, k=1,2, \cdots, n-1}$ which is a transpose matrix of $\left(a_{j k}\right)_{j, k=1,2, \cdots, n-1}$.

Next we study the convexity property concerned with the incident wave front, the reflected one and the obstacle. For $\phi(x)$ with $|\nabla \phi(x)|=1$, we will call the surface $\{y: \phi(y)=\phi(x)\}$ the wave front of $\phi$ passing $x$ (cf. $\S 4$ in [4]). Let $\mathcal{O}$ be an obstacle and fix $x^{0} \in$ $\partial \mathcal{O}$. Let $\phi^{ \pm}$be functions defined in a neighborhood $V$ of $x^{0}$ in $\boldsymbol{R}^{n}$ satisfying

(i) $\left|\nabla \phi^{ \pm}\right|=1$ in $V$,

(ii) $\phi^{+}(x)=\phi^{-}(x)$ for $x \in \partial \mathcal{O} \cap V$,

(iii) $\partial \phi^{+} / \partial \nu>0, \partial \phi^{-} / \partial \nu<0$ on $\partial \mathcal{O} \cap V$.

Let $\{\Phi(\eta) ; \eta \in U\}\left(U \subset \boldsymbol{R}^{n-1}\right)$ be a local coordinate system of $\partial \mathcal{O}$ such that $x^{0}=\Phi(0)$. Assume that the wave front of $\phi^{-}$passing $x^{0}$ has a coordinate representation $\left\{\Psi(\sigma) ; \sigma \in \tilde{U}\left(\tilde{U} \subset \boldsymbol{R}^{n-1}\right)\right\}$ such that $x^{0}=\Psi(0)$. Set $i(\sigma)=\left(\nabla \phi^{-}\right)(\Psi(\sigma))$. Using the implicit function theorem, we define $\eta(\sigma)$ and $l(\sigma)$ which satisfy the following equality 


$$
\Phi(\eta(\sigma))=\Psi(\sigma)+l(\sigma) i(\sigma) \text { for } \sigma \in \tilde{U} \text { (cf. } \S 4 \text { in [4]). }
$$

Then the wave front $\phi^{+}$passing $x^{0}$ is represented of the form

$$
\tau(\sigma)=\Phi(\eta(\sigma))-l(\sigma) r(\sigma)
$$

where

$$
r(\sigma)=i(\sigma)-2\{i(\sigma) \circ \nu(\Phi(\eta(\sigma)))\} \nu(\Phi(\eta(\sigma))) .
$$

Obviously it holds $\left(\nabla \phi^{+}\right)(\tau(\sigma))=r(\sigma)$. Under the above notations, we have the following lemma.

\section{Lemma 2.1。 It holds that}

$$
\begin{gathered}
\left(\partial r / \partial \sigma_{j}(0) \circ \partial \tau / \partial \sigma_{k}(0)\right)_{1 \leqq j, k \leqq n-1}=\left(\partial i / \partial \sigma_{j}(0) \cdot \partial \Psi / \partial \sigma_{k}(0)\right)_{1 \leqq j, k \leqq n-1} \\
+2 \cos \theta^{t}\left(\partial \eta_{j} / \partial \sigma_{k}(0)\right)_{1 \leqq j, k \leqq n-1}\left(\partial \nu \circ \Phi / \partial \eta_{j}(0) \circ \partial \Phi / \partial \eta_{k}(0)\right)_{1 \leqq j, k \leqq n-1} \\
\times\left(\partial \eta_{j} / \partial \sigma_{k}(0)\right)_{1 \leqq j, k \leqq n-1}
\end{gathered}
$$

and that $\operatorname{rank}\left(\partial \eta_{j} / \partial \sigma_{k}(0)\right)_{1 \leqq j, k \leqq n-1}=n-1$, where $\cos \theta=i(0) \cdot\left(-\nu\left(x^{0}\right)\right)$.

Proof. Using $l(0)=0$ and $\left.\nu \cdot\left(\partial \Phi_{\circ} \eta / \partial \sigma_{k}\right)\right|_{\sigma=0}=0$, we have

$$
\begin{aligned}
& \partial r / \partial \sigma_{j}(0) \cdot \partial \tau / \partial \sigma_{k}(0)=\partial i / \partial \sigma_{j}(0) \cdot \partial \Phi_{\circ} \eta / \partial \sigma_{k}(0) \\
& \quad+2 i(0) \cdot\left(-\nu\left(x^{0}\right)\right) \partial \nu \circ \Phi_{\circ} \eta / \partial \sigma_{j}(0) \cdot \partial \Phi_{\circ} \eta / \partial \sigma_{k}(0)
\end{aligned}
$$

Differentiate the both sides of (2.1) with respect to $\sigma_{k}$ and take inner product with $\partial i / \partial \sigma,(0)$. Then we get

$$
\partial i / \partial \sigma_{j}(0) \cdot \partial \Phi \circ \eta / \partial \sigma_{k}(0)=\partial i / \partial \sigma_{j}(0) \cdot \partial \Psi / \partial \sigma_{k}(0) .
$$

Similarly we obtain

$$
\partial \nu / \partial \sigma_{j}(0) \circ \partial \Phi \circ \eta / \partial \sigma_{k}(0)=\sum_{l} \sum_{m} \partial \nu \circ \Phi / \partial \eta_{l} \bullet \partial \Phi /\left.\partial \eta_{m}\left[\partial \eta_{l} / \partial \sigma_{\jmath}\right]\left[\partial \eta_{m} / \partial \sigma_{k}\right]\right|_{\sigma=0}
$$

Combining these equalities imply the equality (2.2). It is easily seen from (2.1) that $\operatorname{rank}\left(\partial \eta_{\jmath} / \partial \sigma_{k}(0)\right)$ is equals to $n-1$. Thus the proof is completed.

Lemma 2. 1 shows that the reflected wave front generates a convex surface when the incident convex wave front hits transversely the convex obstacle. For the strictly convex obstacles $\subset \mathbb{R}^{3}$, Ikawa [4] obtained a relation between the principal curvature of the incident wave front and that of the reflected one.

Let $U$ be a connected open set contained in $\partial \mathcal{O}_{1}$ or $\partial \mathscr{O}_{2}$. Let $\eta(x)$ be a $S^{n-1}$-valued smooth function defined on $U$ satisfying 
$\eta(x) \cdot \nu(x)>0$ for all $x \in U$. We choose $\boldsymbol{R}^{1}$-valued smooth function $l_{U}(x)$ satisfying $\left(v(x)+d\left(l_{U}(x) \eta(x)\right)\right) \cdot v=0$ for all $v \in T_{x} U$, where $d$ denotes the differential. Then the wave front passing $x^{0}+l_{U}\left(x^{0}\right) \eta\left(x^{0}\right)$ $\left(x^{0} \in U\right)$ is the set $\left\{x+l_{U}(x) \eta(x): x \in U\right\}$.

(2.3) This is called the wave front associated with $\eta$.

Let the assumptions in Theorem 2.1 be satisfied. Then we can assume without loss of generality that $\mathcal{O}_{1} \subset\left\{x=\left(x^{1}, \cdots, x^{n}\right) ; x^{1} \leqq 0\right\}$, $\mathcal{O}_{2} \subset\left\{x: x^{1}>0\right\}$ and $\omega=(0, \cdots, 0,1)$. Set

$$
S_{ \pm}^{n-1}=\left\{\theta \in S^{n-1} ; \pm \theta_{1}>0\right\} .
$$

Then, we have

Lemma 2.2. Let $U$ be a connected open set contained in $\partial \mathcal{O}_{1}$. Let $\xi_{0}(x)$ be a $S^{n-1}$-valued smooth function defined on $\bar{U}$ satisfying

(i) $S_{+}^{n-1} \subset\left\{\xi_{0}(x): x \in U\right\}$ and $\left\{\xi_{0}(x): x \in \partial U\right\} \subset \overline{S_{-}^{n-1}}$

$\left(\overline{S_{-}^{n-1}}\right.$ is the closure of $\left.S_{-}^{n-1}\right)$,

(ii) $\xi_{0}(x) \cdot \nu(x)>0$ for all $x \in U$,

(iii) the wave front associated with $\xi_{0}(x)$ is convex surface (cf. (2.3)). We consider the broken ray $L\left(x, \xi_{0}(x)\right)(x \in U)$. Then there exists a connected open set $V$ contained in $\partial \mathcal{O}_{2}$ satisfying

(i)' $\overline{S_{-}^{n-1}} \subset\left\{\xi_{1}\left(x_{1}\right): x_{1} \in V\right\}$ and $\left\{\xi_{1}\left(x_{1}\right): x_{1} \in \partial V\right\} \subset \overline{S_{+}^{n-1}}$,

(ii)' $\xi_{1}\left(x_{1}\right) \cdot \nu\left(x_{1}\right)>0$ for all $x_{1} \in V$,

(iii)' the wave front associated with $\xi_{1}\left(x_{1}\right)$ generates a convex surface.

Proof. Combining Lemma 2.1, with the property (iv) of $A$, the convexity of $\mathrm{\partial O}_{2}$ and the assumption (iii), we see that if above $V$ satisfying (ii)' then $V$ has the property (iii)'. Therefore it suffices to prove the existence of the $V$ with (i)' and (ii)'. The assumption that $\mathcal{O}_{1} \subset\left\{x^{1} \leqq 0\right\}$ and $\mathcal{O}_{2} \subset\left\{x^{1}>0\right\}$ implies that there exists an open

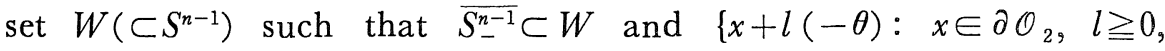
$\theta \in W\} \cap \partial \mathcal{O}_{1}=\phi$. Take an arbitrary $\theta \in W$ and fix it. Let us prove the existence of a broken ray which is reflected in the direction $\xi_{1}=$ $\theta$ by $\mathcal{O}_{2}$. We set

$$
\begin{aligned}
& \xi(y)=-\theta-2\{(-\theta) \cdot \nu(y)\} \nu(y) \quad\left(y \in \partial \mathcal{O}_{2}\right), \\
& \tilde{V}=\left\{y \in \partial \mathcal{O}_{2} ;(-\theta) \cdot \nu(y)<0\right\} .
\end{aligned}
$$


Taking account of Lemma 2.1 and the convexity of $\partial \mathcal{O}_{2}$, we observe that the wave front associated with $\xi(y)$ generates a convex surface. We may assume

$$
\begin{aligned}
& \left\{x+l \xi_{0}(x): \inf l_{U}(x) \leqq l \leqq \sup l_{U}(x), x \in U\right\} \cap \\
& \left\{y+\tilde{l} \xi(y): \inf l_{\tilde{V}}(y) \leqq l \leqq \sup l_{\tilde{V}}(y), y \in \tilde{V}\right\}=\phi .
\end{aligned}
$$

Let $\zeta_{1}=z_{1}+l_{U}\left(z_{1}\right) \xi_{0}\left(z_{1}\right) \quad\left(z_{1} \in \bar{U}\right)$ and $\zeta_{2}=z_{2}+l_{\tilde{V}}\left(z_{2}\right) \xi\left(z_{2}\right) \quad\left(z_{2} \in \overline{\tilde{V}}\right)$ be the points such that

$$
\left|\zeta_{1}-\zeta_{2}\right|=\operatorname{dist} \overline{\left(\left\{x+l_{U}(x) \xi_{0}(x): x \in U\right\}\right.}, \overline{\left.\left\{y+l_{\tilde{V}}(y) \xi(y): y \in \widetilde{V}\right\}\right)} .
$$

If $z_{2}$ belongs to $\partial \tilde{V}$, then $\xi\left(z_{2}\right)$ equals to $-\theta$. But $\theta$ belongs to $W$, hence there cannot exist $\zeta_{1}$, and so we have $z_{2} \in \tilde{V}$. In the same way, we see that $z_{1} \in U$ from the second half of the assumption (i). Therefore the broken ray starting at $z_{1} \in U$ in the direction $\xi_{0}\left(z_{1}\right)$ hits transvarsely at $z_{2} \in V$ and then the direction of the reflection becomes $\theta$. Let $V$ be a set as the collection of $z_{2}$ for each $\theta \in W$. Hence we have been proved the existence of $V$ with $\overline{S_{-}^{n-1}} \subset\left\{\xi_{1}\left(x_{1}\right)\right.$ : $\left.x_{1} \in V\right\}$ in (i)' and (ii)'. Therefore, it only remains to prove $\left\{\xi_{1}\left(x_{1}\right)\right.$ : $\left.x_{1} \in \partial V\right\} \subset \overline{S_{+}^{n-1}}$ in (i). We set $V^{\prime}=\left\{x_{1}: \xi_{1}\left(x_{1}\right) \cdot e \geqq 0\right\} \quad(e=(-1,0, \cdots$, $0))$. It is easily seen that $V^{\prime}$ is a closed connected set. We take an arbitrary $w \in V-V^{\prime}$ and fix it. Since the continuity of $\xi_{1}(y)$ for $y \in V$ follows from the assumption (ii), it suffices to show a inequality $\xi_{1}(w) \cdot e \leqq 0$. If it were $\xi_{1}(w) \cdot e>0$, there should exist $w^{\prime} \in V^{\prime}$ such that $\xi_{1}(w)=\xi_{1}\left(w^{\prime}\right)$. In view of the convexity of the wave front associated with $\xi_{1}$, we can choose a line $x(t)(0 \leqq t \leqq 1)$ joining $w^{\prime}$ and $w$ with $x(0)=w^{\prime}$ and $x(1)=w$ such that $\xi_{1}(x(t))=\xi_{1}(w)=\xi_{1}\left(w^{\prime}\right)$. Consequently we have $\xi_{1}(x(t)) \cdot e>0$ for $0 \leqq t \leqq 1$. According to the choice of $w^{\prime}$ and $w, x(t)$ must intersect $\partial V$. Hence there exists $t_{0}$ $\left(0<t_{0}<1\right)$ such that $\xi_{1}\left(x\left(t_{0}\right)\right) \cdot e=0$. This is a contradiction. Thus the proof is complete.

Remark 2.1. Lemma 2.2 is valid also when $\mathcal{O}_{1}$ and $\mathcal{O}_{2}$ are exchanged each other.

Proof of Theorem 2.1. We take the coordinates given before Lemma 2.2, and consider any broken ray $L\left(x_{0}, \omega\right)$ with $x_{0} \in P$. Assume that the first reflection point $x_{1}$ of $L\left(x_{0}, \omega\right)$ belongs to $\partial \mathcal{O}_{1}$. 
We set

$$
\begin{aligned}
& U=\left\{x \in \partial \mathcal{O}_{1} ; \omega \cdot \nu(x)<0\right\}, \\
& \xi_{0}(x)=\omega-2(\omega \cdot \nu(x)) \nu(x) \quad(x \in U) .
\end{aligned}
$$

Then, taking account of Lemma 2. 1, we see that Lemma 2.2 is satisfied. Using Lemma 2.2 inductively (cf. Remark 2.1) implies that for any positive integer $m(>1)$ there exist at least one broken ray $L\left(x_{0}, \omega\right)$ with ${ }^{\sharp}$ ref $L\left(x_{0}, \omega\right)=m$ such that either $\xi_{m}$ covers $\overline{S_{+}^{n-1}}$ or $\overline{S_{-}^{n-1}}$ when $x_{0}$ moves some open set in $P$. Therefore there exists a broken ray $L\left(x_{0}, \omega\right)$ satisfying the required properties (i) $\sim$ (iv). If there exist the broken rays $L^{i}\left(x_{0}, \omega\right)$ and $L^{i}\left(y_{0}, \omega\right)\left(x_{0} \neq y_{0}\right)$ satisfying (i) (iv), it follows from Lemma 2.1 that the Gaussian curvature of $\partial \Omega$ at $\left\{x_{\jmath}\right\}_{j=1}^{m}$ and $\left\{y_{j}\right\}_{j=1}^{m}$ are zero. Hence the law of reflection gives

$$
\sum_{j=1}^{m+1}\left|x_{j-1}-x_{j}\right|=\sum_{j=1}^{m+1}\left|y_{j-1}-y_{j}\right|
$$

Since the case of $x_{1} \in \partial \mathcal{O}_{2}$ can be treated in the same way, the proof is completed.

\section{Theorem 2.2. If}

$$
\operatorname{dist}\left(\mathcal{O}_{1}, \mathcal{O}_{2}\right)>4 \max _{i=1,2} \operatorname{diam} \mathcal{O}_{i}+C_{1}(\text { cf. (1.4)) }
$$

and

$$
\left|r_{i}(\omega)-r_{2}(\omega)\right|<\max \operatorname{diam} \mathcal{O}_{i}
$$

holds, then we get

$$
\min _{i=1,2} s_{m+1}^{i}>\max _{i=1,2} s_{m}^{i} \quad \text { for } m \geqq 1,
$$

where $s_{m}^{i}$ 's are the ones defined by (1.3).

For the proof of this theorem, we shall prove some lemmas concerned with the reflection points of the broken ray. Set

$$
\begin{array}{r}
\mathscr{L}=\left\{t a_{1}+(1-t) a_{2}: t \in \boldsymbol{R}^{1}, \quad\left(a_{1}, a_{2}\right) \in \partial \mathcal{O}_{1} \times \partial \mathcal{O}_{2},\right. \\
\left.\left|a_{1}-a_{2}\right|=\operatorname{dist}\left(\mathcal{O}_{1}, \mathcal{O}_{2}\right)\right\},
\end{array}
$$

and choose a coordinate system in $\boldsymbol{R}^{n}$ such that $\mathscr{L}_{0}=\{(0, \cdots, 0, l)$ : $\left.l \in \boldsymbol{R}^{1}\right\} \subset \mathscr{L}$.

Lemma 2.3. Take the points $x_{0} \in \partial \mathcal{O}_{1}\left(\operatorname{resp} . \partial \mathcal{O}_{2}\right)$ and $x_{1} \in \partial \mathcal{O}_{2}$ $\left(\right.$ resp. $\left.\partial \mathcal{O}_{1}\right)$ such that 


$$
\operatorname{dist}\left(x_{0}, \mathscr{L}_{0}\right)<\operatorname{dist}\left(x_{1}, \mathscr{L}_{0}\right) \text {. }
$$

Then, for the broken ray $L\left(x_{0},\left(x_{1}-x_{0}\right) /\left|x_{1}-x_{0}\right|\right)$, we have

$$
\operatorname{dist}\left(x_{0}, \mathscr{L}_{0}\right)<\operatorname{dist}\left(x_{1}, \mathscr{L}_{0}\right)<\operatorname{dist}\left(x_{2}, \mathscr{L}_{0}\right)<\operatorname{dist}\left(x_{3}, \mathscr{L}_{0}\right)<\cdots .
$$

Proof. For $x=\left(x^{1}, \cdots, x^{n}\right)$, denote by $x^{\prime}$ the point $\left(x^{1}, \cdots, x^{n-1}, 0\right)$. Let $x(s)=\left(x^{1}(s), \cdots, x^{n}(s)\right)$ be the parametric representation of the broken ray stated above by the length of the broken ray from $x_{0}$ to $x(s)$. Then to prove the lemma, it suffices to show the following inequality.

$$
d\left|x^{\prime}(s)\right| / d s>0 \text { for } s \notin\{0\} \cup\left\{\sum_{k=0}^{j-1} l_{k}: j=1,2, \cdots\right\} .
$$

If $x(s) \in\left\{x_{j}+l_{j} \xi_{j}: 0 \leqq l \leqq l_{j}\right\}$, we have

$$
\begin{aligned}
d\left|x^{\prime}(s)\right| / d s & \geqq\left(\max _{i=1,2} \operatorname{diam} \mathcal{O}_{i}\right)^{-1} \sum_{j=1}^{n-1}\left(x^{j}(s)\right) d x^{j}(s) / d s \\
& =\left(\max _{i=1,2} \operatorname{diam} \mathcal{O}_{i}\right)^{-1}\left\{x_{j}-\left(s-\sum_{k=0}^{j-1} l_{k}\right) \xi_{j}\right\}^{\prime} \circ \xi_{j} \\
& \geqq\left(\max _{i=1,2} \operatorname{diam} \mathcal{O}_{i}\right)^{-1} x_{j}^{\prime} \cdot \xi_{j \bullet}
\end{aligned}
$$

On the other hand, we get

$$
x_{j}^{\prime} \cdot \xi_{j}=x_{j}^{\prime} \cdot \xi_{j-1}+2\left(-\nu\left(x_{j}\right) \cdot \xi_{j-1}\right) \nu\left(x_{j}\right) \cdot x_{j}^{\prime} \text { 。 }
$$

Since for some constant $\rho>0$

$$
-\nu\left(x_{j}\right) \cdot \xi_{j-1}>\rho \text { for any } j(\text { Lemma } 3.1 \text { in }[4]),
$$

we immediately have

$$
\begin{aligned}
x_{j}^{\prime} \cdot \xi_{j} & \geqq x_{j}^{\prime} \circ \xi_{j-1}+2 \rho \nu\left(x_{j}\right) \circ x_{j}^{\prime} . \\
& \geqq x_{j-1}^{\prime} \cdot \xi_{j-1}+2 \rho \nu\left(x_{j}\right) \cdot x_{j}^{\prime} .
\end{aligned}
$$

This, combined with (2.5), implies

$$
\begin{aligned}
d\left|x^{\prime}(s)\right| / d s & \geqq\left(\max _{i=1,2} \operatorname{diam} \mathcal{O}_{i}\right)^{-1}\left\{x_{1}^{\prime} \cdot \xi_{1}+2 \rho \sum_{k=2}^{j} \nu\left(x_{k}\right) \cdot x_{k}^{\prime}\right\} \\
& \geqq\left(\max _{i=1,2} \operatorname{diam} \mathcal{O}_{i}\right)^{-1}\left\{x_{1}^{\prime} \bullet \xi_{0}+2 \rho \sum_{k=1}^{j} \nu\left(x_{k}\right) \cdot x_{k}^{\prime}\right\} .
\end{aligned}
$$

From the assumption that $\operatorname{dist}\left(x_{0}, \mathscr{L}_{0}\right)<\operatorname{dist}\left(x_{1}, \mathscr{L}_{0}\right)$, it follows that

$$
x_{1}^{\prime} \cdot \xi_{0}>0 \text { and } \nu\left(x_{k}\right) \circ x_{k}^{\prime}>0 \text { for } k \geqq 1 \text {. }
$$

so finally we have

$$
d\left|x^{\prime}(s)\right| / d s>0
$$

which proves our lemma。

Remark 2.2. Even if $x_{0}$ does not belong to $\mathscr{L} \cap \partial \Omega$, Lemma 2.2 is also true under the assumption that $\operatorname{dist}\left(x_{0}, \mathscr{L}_{0}\right) \leqq \operatorname{dist}\left(x_{1}, \mathscr{L}_{0}\right)$. 
Lemma 2.4. Suppose that " $\mathrm{ref} L^{1}\left(x_{0}, \omega\right)={ }^{\#} \mathrm{ref} L^{2}\left(x_{0}, \omega\right)=2 m-1$, and let $x_{1}, \cdots, x_{2 m-1}$ be the reflection points of the broken ray $L^{1}\left(x_{0}, \omega\right)$ or $L^{2}\left(x_{0}, \omega\right)$ in Theorem 2.1. Then there holds that

$$
x_{j}=x_{2 m-1-(j-1)} \quad \text { for } j=1,2, \cdots, m,
$$

and that

$$
\operatorname{dist}\left(x_{1}, \mathscr{L}_{0}\right)>\operatorname{dist}\left(x_{2}, \mathscr{L}_{0}\right)>\cdots>\operatorname{dist}\left(x_{m}, \mathscr{L}_{0}\right)
$$

Proof. If $x_{1} \neq x_{2 m-1}$, then $x_{2} \neq x_{2 m-2}$ from Lemma 2.1 and $\xi_{2 m-1}=$ $-\omega$. Therefore successively we obtain $x_{m} \neq x_{2 m-m}\left(=x_{m}\right)$, which is a contradiction. So we have

$$
x_{\jmath}=x_{2 m-1-(j-1)} \quad \text { for } j=1,2, \cdots, m .
$$

Let $\operatorname{dist}\left(x_{i}, \mathscr{L}_{0}\right) \leqq \operatorname{dist}\left(x_{\imath+1}, \mathscr{L}_{0}\right)$ for an $i(1 \leqq i \leqq m-2)$. Then by Lemma 2.3 and Remark 2.2, we have

$$
\operatorname{dist}\left(x_{j+1}, \mathscr{L}_{0}\right)<\operatorname{dist}\left(x_{j+2}, \mathscr{L}_{0}\right) \quad \text { for all } i \leq j .
$$

However, from (2.7) follows the equality $\operatorname{dist}\left(x_{m-1}, \mathscr{L}_{0}\right)=\operatorname{dist}\left(x_{m+1}\right.$, $\left.\mathscr{L}_{0}\right)$, which is not consistent with (2.8). Hence we arrive at (2.6). In case "ref $L$ is even, the following lemma is obtained by the same procedures as in Lemma 2. 4.

Lemma 2.5. Suppose that ${ }^{\sharp} \mathrm{ref} L^{1}\left(x_{0}, \omega\right)={ }^{\sharp} \mathrm{ref} L\left(x_{0}, \omega\right)=2 m$, and let $x_{1}, \cdots, x_{2 m}$ be the reflection points of the broken ray $L^{1}\left(x_{0}, \omega\right)$ or $L^{2}\left(x_{0}, \omega\right)$ in Theorem 2.1. Then there exists the only integer $l$ such that

$$
\begin{aligned}
& \operatorname{dist}\left(x_{1}, \mathscr{L}_{0}\right)>\operatorname{dist}\left(x_{2}, \mathscr{L}_{0}\right)>\cdots>\operatorname{dist}\left(x_{l}, \mathscr{L}_{0}\right), \\
& \operatorname{dist}\left(x_{l}, \mathscr{L}_{0}\right)<\operatorname{dist}\left(x_{l+1}, \mathscr{L}_{0}\right)<\cdots<\operatorname{dist}\left(x_{2 m}, \mathscr{L}_{0}\right) .
\end{aligned}
$$

Lemma 2.6. For any positive integer $m$, let $\left\{x_{\jmath}\right\}_{j=1}^{m}$ be the reflection points of the broken ray such that

$$
\operatorname{dist}\left(x_{1}, \mathscr{L}_{0}\right)>\operatorname{dist}\left(x_{2}, \mathscr{L}_{0}\right)>\cdots>\operatorname{dist}\left(x_{m}, \mathscr{L}_{0}\right) .
$$

Then there exists a constant $\tilde{G}_{1}>0$ independent of $m$ such that

$$
\sum_{j=1}^{m-1} l_{\jmath}-(m-1) \operatorname{dist}\left(\mathcal{O}_{1}, \mathcal{O}_{2}\right)<\tilde{G}_{1} \text {. }
$$

Proof of Lemma 2.6. Without loss of generality we may assume that $0 \in \mathscr{L}_{0} \cap \partial \mathcal{O}_{1}, \mathcal{O}_{1} \subset\left\{x^{n} \leqq 0\right\}$ and $\mathcal{O}_{2} \subset\left\{x^{n}>0\right\}$. Let

$$
\Pi_{1}=\left\{x: x=\left(x^{\prime}, 0\right)\right\} \text {, }
$$




$$
\Pi_{2}=\Pi_{1}+\left(0, \cdots, 0, \operatorname{dist}\left(\mathcal{O}_{1}, \mathcal{O}_{2}\right)\right) .
$$

For simplicity, we set $\tilde{d}=\operatorname{dist}\left(\mathcal{O}_{1}, \mathcal{O}_{2}\right)$.

Since $\operatorname{dist}\left(x_{j}, \mathscr{L}_{0}\right)>\operatorname{dist}\left(x_{j+1}, \mathscr{L}_{0}\right)$, we have

$$
\begin{aligned}
& l_{j}-\operatorname{dist}\left(\mathcal{O}_{1}, \mathcal{O}_{2}\right) \\
& =l_{j}\left|\xi_{j}^{\prime}\right|^{2}+l_{j}\left(\xi_{j}^{n}\right)^{2}-\tilde{d} \\
& \leqq l_{j}\left|\xi_{j}^{\prime}\right|^{2}+l_{j}\left|\xi_{j}^{n}\right|-\tilde{d} \\
& \leqq \begin{cases}l_{j}\left|\xi_{j}\right|^{2}+\operatorname{dist}\left(x_{j}, \Pi_{1}\right)+\operatorname{dist}\left(x_{j+1}, \Pi_{2}\right) & \text { if } x_{j} \in \partial \mathcal{O}_{1} \\
& \text { and } x_{j+1} \in \partial \mathcal{O}_{2}, \\
l_{j}\left|\xi_{j}^{\prime}\right|^{2}+\operatorname{dist}\left(x_{j}, \Pi_{2}\right)+\operatorname{dist}\left(x_{j+1}, \Pi_{1}\right) & \text { if } x_{j} \in \partial \mathcal{O}_{2} \\
& \text { and } x_{j+1} \in \partial \mathcal{O}_{1} .\end{cases}
\end{aligned}
$$

There exists a constant $C_{2}>0$ such that

$$
\operatorname{dist}\left(x_{j}, \Pi_{1}\right)+\operatorname{dist}\left(x_{j+1}, \Pi_{2}\right) \leqq C_{2}\left\{\nu\left(x_{\jmath}\right) \cdot x_{j}^{\prime}+\nu\left(x_{j+1}\right) \cdot x_{j+1}^{\prime}\right\} \text { for all } j \text {. }
$$

Hence we obtain

$$
\begin{aligned}
& \sum_{j=1}^{m-1} l_{j}-(m-1) \tilde{d} \\
\leqq & \sum_{j=1}^{m-1} l_{j}\left|\xi_{j}^{\prime}\right|_{j}^{2}+2 C_{2} \sum_{j=1}^{m-1} \nu\left(x_{\jmath}\right) \circ x_{j}^{\prime} .
\end{aligned}
$$

On the other hand, by the same procedures as in the proof of Lemma 2. 3, we obtain

$$
\begin{aligned}
x_{\jmath}^{\prime} \cdot \xi_{J} & \geqq x_{j-1}^{\prime} \cdot \xi_{j-1}^{\prime}+l_{J-1}\left|\xi_{j-1}^{\prime}\right|^{2}+2 \rho \nu\left(x_{\jmath}\right) \cdot x_{j}^{\prime} \\
& \geqq x_{1}^{\prime} \cdot \xi_{1}^{\prime}+\sum_{k=1}^{j-1} l_{k}\left|\xi_{k}^{\prime}\right|^{2}+2 \rho \sum_{k=1}^{\prime} \nu\left(x_{k}\right) \cdot x_{k}^{\prime} \\
& \geqq-\max _{i=1,2} \operatorname{diam} \mathcal{O}_{i}+\sum_{k=1}^{j-1} l_{k}\left|\xi_{k}^{\prime}\right|^{2}+2 \rho \sum_{k=1}^{j} \nu\left(x_{k}\right) \cdot x_{k}^{\prime} \text { for all } j .
\end{aligned}
$$

From the assumption $\operatorname{dist}\left(x_{j}, \mathscr{L}_{0}\right)>\operatorname{dist}\left(x_{j+1}, \mathscr{L}_{0}\right)$, we have $x_{j}^{\prime} \cdot \xi_{j} \leqq 0$. This, combined with the above estimates, gives

$$
\max _{i=1,2} \operatorname{diam} \mathcal{O}_{i} \geqq \sum_{k=1}^{j-1} l_{k}\left|\xi_{k}^{\prime}\right|^{2}+2 \rho \sum_{k=1}^{j} \nu\left(x_{k}\right) \cdot x_{k}^{\prime} .
$$

Therefore, we conclude from (2.10) that

$$
\begin{aligned}
& \sum_{j=1}^{m-1} l_{j}-(m-1) \tilde{d} \\
& \leqq\left(1+2 C_{2} \rho^{-1}\right) \max _{i=1,2} \operatorname{diam} \mathcal{O}_{i} .
\end{aligned}
$$

Then for $\tilde{C}_{1}=1+2 C_{2} \rho^{-1}$, we have (2.9).

Now we are in position to give

Proof of Theorem 2.2. Let $x_{0}, \cdots, x_{m+1}$ be the points associated with $\max _{i=1,2} s_{m}^{i}$ (cf. (1.3)). Then we have

$$
\min _{i=1,2} s_{m+1}^{i} \geqq m \operatorname{dist}\left(\mathcal{O}_{1}, \mathcal{O}_{2}\right) \text {, }
$$


and

$$
\begin{aligned}
\max _{i=1,2} s_{m}^{i} & \leqq\left|x_{0}-x_{1}\right|+\left|x_{m}-x_{m+1}\right|-2+\sum_{j=1}^{m-1} l_{\jmath} \\
& \leqq 2 \max \operatorname{diam} \mathcal{O}_{i}+2\left|r_{1}(\omega)-r_{2}(\omega)\right|+\sum_{\jmath=1}^{m-1} l_{j} \\
& \leqq 4 \max \operatorname{diam} \mathcal{O}_{i}+\sum_{\jmath=1}^{m-1} l_{\jmath} .
\end{aligned}
$$

Thus from Lemma 2.4, 2.5, 2.6, it follows that there exists a constant $C_{1}>0$ independent of $m$ such that

$$
(0 \leqq) \quad \sum_{j=1}^{m-1} l,-(m-1) \operatorname{dist}\left(\mathcal{O}_{1}, \mathcal{O}_{2}\right)<C_{1}
$$

and hence

$$
\max _{i=1,2} s_{m}^{i} \leqq 4 \max \operatorname{diam} \mathcal{O}_{i}+C_{1}+(m-1) \operatorname{dist}\left(\mathcal{O}_{1}, \mathcal{O}_{2}\right) .
$$

From the assumption that $\operatorname{dist}\left(\mathcal{O}_{1}, \mathcal{O}_{2}\right)>4 \max \operatorname{diam} \mathcal{O}_{i}+C_{1}$, we have the inequality

$$
\max _{i=1,2} s_{m}^{i}<\min _{i=1,2} s_{m+1}^{i},
$$

which completes our proof.

Remark 2.3. Taking account of the above inequality (2.11), we have proved (1.4) in Theorem 2.

The following theorem is concerned with the distribution of $s_{m}^{i}$ defined by (1.3) as $m \rightarrow+\infty$.

Theorem 2.3. Assume that $\operatorname{dist}\left(\mathcal{O}_{1}, \mathcal{O}_{2}\right)=\tilde{d}>d_{0}\left(d_{0}\right.$ is the constant in Theorem 2.2) and that $\omega \in S^{n-1}$ satisfy the assumption stated in Theorem 1. Moreover suppose that there exists the only one pair of points $\left(a_{1}, a_{2}\right) \in \partial \mathcal{O}_{1} \times \partial \mathcal{O}_{2}$ such that $\left|a_{1}-a_{2}\right|=\tilde{d}$ then we have

(i) $\lim _{m \rightarrow+\infty}\left(s_{m+1}^{i}-s_{m-1}^{i}\right)=2 \tilde{d} \quad(i=1,2)$,

(ii) $\lim _{m \rightarrow+\infty}\left\{s_{2 m}^{i}-2^{-1}\left(s_{2 m-1}^{1}+s_{2 m-1}^{2}\right)\right\}=\tilde{d}(i=1,2)$.

For the proof, the following lemma is needed.

Lemma 2. 7 (Lemma 3.3 in Ikawa [4]). Set

$$
U(\delta)=\{x \in \partial \Omega ; \operatorname{dist}(x, \mathscr{L}) \leqq \delta\}, \delta>0 \text { (for } \mathscr{L}, \text { see }(2.4)) .
$$

Let $x_{1}, x_{2}, \cdots$ be the reflection points of a broken ray $L\left(x_{0}, \xi_{0}\right)$ and assume that $x_{1} \in \partial \Omega-U(\delta)$ and $L\left(x_{0}, \xi_{0}\right) \cap U(\delta)=\phi$. Then there exists a positive constant $C_{\delta}$ such that 
"ref $L\left(x_{0}, \xi_{0}\right) \leqq C_{\delta}$.

Remark 2.4. Ikawa [4] assume that $\mathcal{O}_{i}$ is strictly convex. But it can be easily checked that we can apply the proof of Lemma in [4] to our case.

Proof of Theorem 2.3. First, let us show that for any $\varepsilon>0$

$$
\left|\tilde{d}+2^{-1}\left(s_{2 m-1}^{i}-s_{2 m+1}^{i}\right)\right|<\varepsilon
$$

if $m$ is sufficiently large. Combining this with (ii) in the theorem, we get (i) in our theorem. We take $\delta$ in Lemma 2.7 so that $\delta=\varepsilon$. Let $\left\{x_{\jmath}\right\}_{j=0}^{2 m}$ and $\left\{y_{\jmath}\right\}_{j=0}^{2 m+2}$ be the points defining $s_{2 m-1}^{i}$ and $s_{2 m+1}^{i}$ respectively (cf. (1.3)). Since the equalities $x_{j}=x_{2 m-1-(j-1)}(j=1,2, \cdots$, $m$ ) follow from Lemma 2.4, we have

$$
2^{-1} s_{2 m-1}^{i}=\sum_{j=0}^{m-1}\left|x_{j}-x_{j+1}\right|-1 \text {. }
$$

From Lemma 2.7, there exists a positive integer $l=l(\varepsilon)$ independent of $m$ such that $x_{j}(1 \leqq j \leqq m-1)$ does not belong to $U(\varepsilon)$ for $j \leqq l$. Also we have the same properties for $\left\{y_{j}\right\}_{j=1}^{m+1}$. Hence we have

$$
\begin{aligned}
& \left|\tilde{d}+2^{-1}\left(s_{2 m-1}^{i}-s_{2 m+1}^{i}\right)\right| \\
\leqq & \left|\sum_{J=0}^{l-1}\left(\left|x_{\jmath}-x_{\jmath+1}\right|-\left|y_{\jmath}-y_{\jmath+1}\right|\right)\right| \\
+ & \mid \sum_{j=l}^{m-1}\left(\left|x_{\jmath}-x_{\jmath+1}\right|-\left|y_{\jmath}-y_{\jmath+1}\right| \mid\right. \\
+ & |\tilde{d}-| y_{m}-y_{m+1}|| \\
\equiv & I_{1}+I_{2}+I_{3} .
\end{aligned}
$$

As in the proof of Lemma 2.6, we obtain

$$
\begin{aligned}
& \{m-1-(l-1)\} \tilde{d} \\
\leqq & \sum_{j=l}^{m-1}\left|x_{\jmath}-x_{\jmath+1}\right| \\
\leqq & \{m-1-(l-1)\} \tilde{d}+C(\varepsilon),
\end{aligned}
$$

where the constant $C(\varepsilon)(>0)$ does not depend on $m$ and tends to 0 as $\varepsilon \rightarrow 0$. The same inequality holds for $\sum_{j=l}^{m-1}\left|y_{j}-y_{\jmath+1}\right|$, too. Therefore we obtain

$$
I_{2} \leqq 2 C(\varepsilon) \text {. }
$$

From the assumptions, $\mathscr{L}$ consists of the only one line. Taking account of this result, Lemma 2.2 and Lemma 2.4, we have

$$
I_{1}+I_{3}<\varepsilon \text { as } m \rightarrow+\infty \text {. }
$$

which gives the required inequality. 
Let us prove (ii). Let $\left\{x^{i}\right\}_{j=0}^{2 m}$ and $\left\{y_{j}^{i}\right\}_{j=0}^{2 m+1}$ be the sets of points defining $s_{2 m-1}^{i}$ and $s_{2 m}^{i}(i=1,2)$ respectively. From Theorem 2.1 it follows that $s_{2 m}^{1}=s_{2 m}^{2}$. Hence we get

$$
\begin{aligned}
& \left|s_{2 m}^{2}-2^{-1}\left(s_{2 m-1}^{1}+s_{2 m-1}^{2}\right)-\tilde{d}\right| \\
= & \left|s_{2 m}^{1}-2^{-1}\left(s_{2 m-1}^{1}+s_{2 m-1}^{2}\right)-\tilde{d}\right| \\
\leqq & \left.\left|\sum_{j=0}^{m-1}\left(\left|y_{j}^{1}-y_{j+1}^{1}\right|-\left|x_{j}^{1}-x_{j+1}^{1}\right|\right)+\right|\left|y_{m}^{1}-y_{m+1}^{1}\right|-\tilde{d}\right) \mid \\
+ & \mid \sum_{j=m+1}^{2 m}\left(\left|y_{\jmath}^{1}-y_{\jmath+1}^{1}\right|-\left|x_{j}^{2}-x_{j+1}^{2}\right|\right) \\
\equiv & \tilde{I}_{1}+\tilde{I}_{2}+\tilde{I}_{3} .
\end{aligned}
$$

By the same arguments as above, we see that $\tilde{I}_{i} \rightarrow 0 \quad(i=1,2,3)$ as $m \rightarrow+\infty$. Hence (ii) is obtained. Thus our theorem has been proved.

\section{§3. A Sketch of the Procedures in the Proof of Main Theorems}

In view of Theorem 2. 1, 2.2, 2.3 and Remark 2.3, all we want to do is to prove Theorem 1 and (ii) in Theorem 2. The procedure of these proofs are the same as those used in [10]. Therefore we only sketch it here (for details procedures, see $\S 3$ in [10]). To analyze the singularities of $S(\cdot,-\omega, \omega)$, we use the following representation (cf. Majda [8] and Soga [14]):

$$
\begin{aligned}
S(s, \theta, \omega)=\int_{\partial \Omega}\left\{\nu \cdot \theta \partial_{t}^{n-1} v(x \cdot \theta-s, x ; \omega)\right. & \\
& \left.-\partial_{t}^{n-2} \partial_{\nu} v(x \cdot \theta-s, x ; \omega)\right\} d S_{x} \quad(\theta \neq \omega),
\end{aligned}
$$

where $v(t, x ; \omega)$ is the solution of the mixed problem

$$
\begin{cases}\square v(t, x ; \omega)=0 \quad \text { in } \boldsymbol{R}^{1} \times \Omega, & \\ v=2^{-1}(-2 \pi i)^{1-n} \delta(t-x \cdot \omega) & \text { on } \boldsymbol{R}^{1} \times \partial \Omega, \\ v=0 & \text { for } t<r(\omega) .\end{cases}
$$

Taking account of the relation between the singularities of $v$ and the broken rays of the geometrical optics considered in Guillemin [2], Petkov [11], etc., we may expect that sing supp $S(\cdot,-\omega, \omega)$ is contributed only by the broken rays associated with (1.3). We can construct the asymptotic solution of the equation (3.2) near these broken rays in the same way as in $\S 7$ in [4], and reduce the proof of Theorem 1 and (ii) in Theorem 2 to showing that the following integral does not decrease rapidly as $|\sigma| \rightarrow \infty$ : 


$$
\int_{\partial \Omega} e^{i \sigma\left(x \cdot \omega+\phi_{m}^{\jmath}(x)\right)} \alpha\left(-x \cdot \omega-\phi_{m}^{j}(x)+2 \min _{i=1,2} r_{i}(\omega)+s_{m}^{i}\right) \beta(x) d S_{x}
$$

where $\beta(x)$ is a non-zero $C^{\infty}$-function, $\alpha(s)$ is a cut-off $C^{\infty}$-function with small support and satisfying $\alpha(0) \neq 0$ and $\phi_{m}^{j}$ is a phase function associated with the broken ray defining $s_{m}^{j}$. By the results obtained by Soga [15] when $\mathcal{O}_{i}$ is convex, we observe that (3.3) does not decrease rapidly as $|\sigma| \rightarrow \infty$.

\section{Acknowledgements}

The author would like to thank Professor Hideo Soga for his helpful discussion and Professor Takao Kakita for his continuous encouragement. He would also like to thank the referee for his valuable advice.

\section{References}

[1] Flanders, H., Differential forms, Academic Press, (1963).

[2] Guillemin, V., Sojourn time and asymptotic properties of the scattering matrix, Publ. RIMS, Kyoto Univ., 12 Suppl. (1977), 69-88.

[3] Hörmander, L., Fourier integral operators I, Acta Math., 127 (1971), 79-183.

[4] Ikawa, M., Decay of solutions of the wave equation in the exterior of two convex obstacles, Osaka J. Math., 19 (1982)., 459-509.

[5] Kumano-go, H., Pseudo-differential operators, MIT Press, (1982).

[6] Lax, P.D. and Phillips, R. S., Scattering Theory, Academic Press, (1967).

[7] Scattering theory for the acoustic equation in an even number of space dimensions, Indiana Univ. Math. J., 22 (1972), 101-134.

[8] Majda, A., A representation formula for the scattering operator and the inverse problem for arbitrary bodies, Comm. Pure Apple. Math., 30 (1977), 165-194.

[9] Matsumura, M., Asymptotic behavior at infinity for Green's function of first order systems with characteristics of nonuniform multiplicity, Publ. RIMS, Kyoto Univ., 12 (1976), 313-377.

[10] Nakamura, S. and Soga, H., Singularities of the scattering kernel for two balls, $J$. Math. Soc. Japan., 40 (1988), 205-220.

[11] Petkov, V.M., High frequency asymptotic of the scattering amplitude for non-convex bodies, Comm. in Partial differential Equations, 5 (1980), 293-329.

[12] Petkov, V.M. and Stojanov, L. N., Singularities of the scattering kernel for non convex obstacles, to appear.

[13] Soga, H., Oscillatory integrals with degenerate stationary points and their applications to the scattering theory, Comm. in Partial Differential Equations, 6 (1981), 273-287.

[14] Singularities of the scattering kernel for convex obstacles, J. Math. Kyoto Univ., 22 (1983), 729-765.

[15] Conditions against rapid decrease of oscillatory integrals and their applications to inverse scattering problems, Osaka J.Math., 23 (1986), 441-456.

[16] Taylor, M. E., Grazing rays and reflection of singularities of solutions to wave equations, Comm. Pure Appl. Math., 29 (1976), 1-38.

[17] Yamamoto, K., Characterization of a convex obstacle by singularities of the scattering kernel, J. Differential Equations, 64 (1986), 283-293. 